\title{
Parallel Execution of Hash Joins in Parallel Databases
}

\author{
Hui-I Hsiao, Ming-Syan Chen, Senior Member, IEEE, and Philip S. Yu, Fellow, IEEE
}

\begin{abstract}
In this paper, we explore two important issues, processor allocation and the use of hash filters, to improve the parallel execution of hash joins. To exploit the opportunity of pipelining for hash join execution, a scheme to transform a bushy execution tree to an allocation tree is first devised. In an allocation tree, each node denotes a pipeline. Then, using the concept of synchronous execution time, processors are allocated to the nodes in the allocation tree in such a way that inner relations in a pipeline can be made available at approximately the same time. Also, the approach of hash filtering is investigated to further improve the parallel execution of hash joins. Extensive performance studies are conducted via simulation to demonstrate the importance of processor allocation and to evaluate various schemes using hash filters. It is experimentally shown that processor allocation is, in general ,the dominant factor to performance, and the effect of hash filtering becomes more prominent as the number of relations in a query increases.
\end{abstract}

Index Terms-Hash filters, pipelining, bushy trees, hash joins.

\section{INTRODUCTION}

$I^{N}$ $\mathrm{N}$ recent years, parallel database machines have drawn a considerable amount of attention from both the academic and industrial communities due to their high potential for parallel execution of complex database operations [1], [3], [8], [11], [17], [32]. In relational database systems, joins are the most expensive operations to execute, especially with the increases in database size and query complexity [23], [38]. As a result, it has become imperative to develop solutions for efficient execution of multijoin queries for future database management [9], [13], [20], [25].

A query plan is usually compiled into a tree of operators, called a join sequence tree, where a leaf node represents an input relation and an internal node represents the resulting relation from joining the two relations associated with its two child nodes. There are three categories of query trees: left-deep trees, right-deep trees, and bushy trees, where left-deep and right-deep trees are also called linear execution trees, or sequential join sequences. Examples of the three forms of query trees are shown in Fig. 1. A significant amount of research efforts has been elaborated upon developing join sequences to improve the query execution time. The work reported in [30] was among the first to explore sequential join sequences, and there have been several results reported for sequential join sequences [14], [18], [33], [34]. The bushy tree join sequences, on the other hand, did not attract as much attention as sequential ones until very recently. This can be explained, in part, by the reasons that, in the past, the power/size of a multiprocessor system was

- H.-I. Hsaio and P.S. Yu are with the IBM T.J. Watson Research Center, P.O. Box 704, Yorktown Heights, NY 10598.

E-mail: \{hhsiao,psyu\}@watson.ibm.com.

- M.-S. Chen is with the Electrical Engineering Department, National Taiwan University, Taipei, Taiwan, Republic of China.

E-mail:mschen@cc.ee.ntu.edu.tw.

Manuscript received 12 Aug. 1996

For information on obtaining reprints of this article, please send e-mail to: transpds@computer.org, and reference IEEECS Log Number 100271.0. limited, and that the query structure used to be too simple to require further parallelizing as a bushy tree. It is noted, however, that these two limiting factors have been phased out by the rapid increase in the capacity of multiprocessors and the trend for queries to become more complicated nowadays [38], thereby justifying the necessity of exploiting bushy trees. As a result, it has attracted an increasing amount of attention to explore the use of bushy trees for parallel query processing. A combination of analytical and experimental results was given in [16], [31] to shed some light on the complexity of choosing left-deep and bushy trees. An integrated approach dealing with both intraoperator and interoperator parallelism was presented in [21], where a greedy scheme taking various join methods and their corresponding costs into consideration was proposed. A two-step approach to deal with join sequence scheduling and processor allocation for parallel query processing was devised in [6]. Several query plans in processing multijoin queries in a shared-nothing architecture were investigated in [27]. In addition, experimental studies on evaluating various query plan generation strategies were conducted in [36].

Among various join methods, the hash join has been the focus of much research effort and reported to have performance superior to that of others, particularly because it presents an opportunity for pipelining [7], [12], [22], [24],

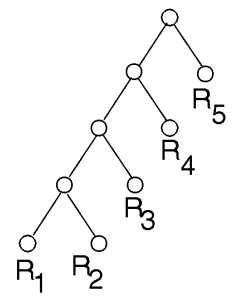

(a) left-deep tree

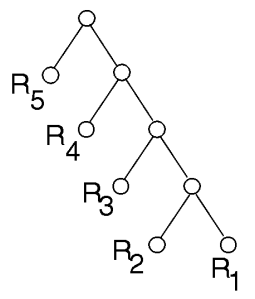

(b) right-deep tree

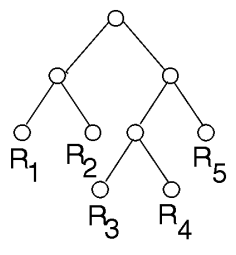

(c) bushy tree
Fig. 1. Illustration of different query trees. 
[26], [35]. A pipeline of hash joins is composed of several stages, each of which is associated with one join operation that can be executed, in parallel, by several processors. Though pipelining has been shown to be very effective in reducing the query execution time, prior studies on pipelined hash joins have focused mainly on heuristic methods for query plan generation. Most of the prior work on query plan generation, such as static right-deep scheduling, dynamic bottom-up scheduling [29], and segmented rightdeep trees [5], resorted to simple heuristics to allocate processors to pipeline stages. Also, due to the shared-nothing architecture assumed, prior methods mainly dealt with memory as a constraint for the execution of pipelined hash joins. Little effort was made to take processing power into consideration and optimize processor allocation. It has been shown that, for sort-merge joins, the execution of bushy trees can outperform that of linear trees, especially when the number of relations in a query is large [6]. However, as far as the hash join is concerned, the scheduling for an execution plan of a bushy tree structure is much more complicated than that of a right-deep tree structure. Particularly, it is very difficult to achieve the synchronization required for the execution of bushy trees such that the effect of pipelining can be fully utilized. This is the very reason that most prior studies on pipelined hash joins focused on the use of right-deep trees.

In this paper, we explore two important issues, processor allocation and the use of hash filters, to improve the parallel execution of hash joins. Clearly, to execute a given query, processors should be allocated in such a way that the query execution time is minimized. Also, the technique of hash filtering can be employed to further reduce the query execution time. Note that to exploit the opportunity of pipelining for hash join execution, one would naturally like to identify and execute a group of hash joins in the bushy tree in a way of pipelining. However, it can be seen that such regrouping of joins, while taking advantage of pipelining, makes the execution dependency ${ }^{2}$ in the bushy tree intractable, which, in turn, causes the problem of processor allocation to be much more complicated. To remedy this, we first devise in this paper a scheme to transform a bushy execution tree to an allocation tree in which each node denotes a pipeline. Then, using the concept of synchronous execution time [6], processors are allocated to the nodes in the allocation tree in such a way that inner relations in a pipeline can be made available at approximately the same time, thus solving the execution dependency for the parallel execution of pipelined hash joins. In addition, the approach of hash filtering is investigated to improve the query execution time. Note that depending on the cost and the benefit of hash filters, there are various schemes to determine the hash filter generation. Extensive performance studies via a detailed simulation are conducted to demonstrate the importance of processor allocation and to evaluate different schemes using hash filters. Among all schemes for hash filtering evaluated, the one to build hash filters for inner relations only emerges as a winner. It is experimentally

1. A study on using hash filters to improve the execution of sort-merge joins can be found in [4]

2. Execution dependency means that a pipeline cannot be performed until all of its input relations are available. shown that processor allocation is in general the dominant factor to performance, and the effect of hash filtering becomes more prominent as the number of relations in a query increases.

The rest of this paper is organized as follows. Preliminaries are given in Section 2. Schemes for processor allocation and hash filtering are described in Section 3. Performance studies on various schemes are conducted in Section 4 via simulation. This paper concludes with Section 5 .

\section{Preliminaries}

\subsection{Notation and Assumptions}

We assume that a query is of the form of conjunctions of equi-join predicates. We use $\left|R_{i}\right|$ to denote the cardinality of a relation $R_{i}$ and $|A|$ to denote the cardinality of the domain of an attribute $A$. As in most prior work on the execution of database operations, we assume that the execution time incurred is the primary cost measure for the processing of database operations. Also, we focus on the execution of complex queries [33], i.e., queries involving many relations. Notice that such complex queries can become frequent in real applications due to the use of views [38]. The architecture assumed in the performance study in Section 4 is a multiprocessor system with distributed memories and shared disks containing database data.

A pipeline of hash joins is composed of several stages, each of which is associated with one join operation. The relation in a hash join that is loaded into memory to build the hash table is called the inner relation, while the other relation, whose tuples are used to probe the hash table, is called the outer relation. The inner relations of a pipeline are the inner relations of its stages. The outer relation of a pipeline is defined to be the outer relation of its first stage. The execution of a pipeline of hash joins consists of two phases:

1) the table-building phase, and

2) the tuple-probing phase.

In the table-building phase, the hash tables of the inner relations are built using hash functions on join attributes. In the tuple-probing phase, tuples of the outer relation are first probed, one by one, against the entries in the hash table of the first stage using the corresponding hash function. If there are matches, the resulting tuples are generated, and then sent to the next stage for similar processing. Pipelining has two major advantages. First, I/O costs are significantly reduced, since only the output tuples of the last join would need to be written to disk. The output relations of the intermediate joins need not even exist as whole tables in memory. Second, the first tuples of the resulting relation (i.e., the output relation of the last join) can be produced earlier, not only reducing the response time perceived by the end user, but also enabling application programs to start processing query results earlier. The execution of one pipeline is given in Fig. 2 for illustration, where nine processors are allocated to execute a pipeline of three stages.

Both CPU and I/O costs of executing a query are considered in this paper. CPU cost is determined by the pathlength, i.e., the total number of tuples processed multiplied by the 


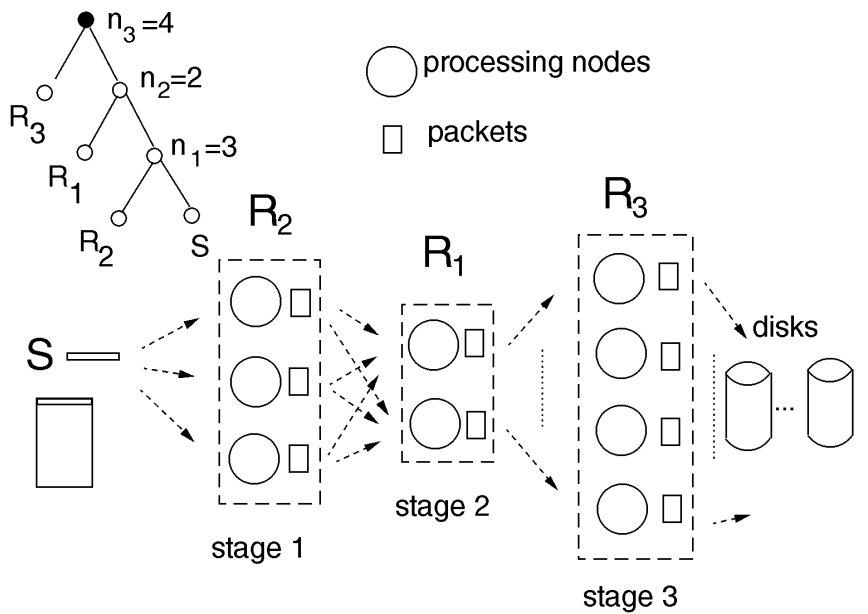

Fig. 2. Execution of one pipeline.

number of $\mathrm{CPU}$ instructions required for processing each tuple. A parameter on CPU speed (i.e., MIPS) is used to compute the CPU processing time from the number of CPU instructions incurred. $\mathrm{I} / \mathrm{O}$ cost for processing a query is determined by disk service time per page multiplied by the total number of page I/Os. By doing such, we can appropriately vary the $\mathrm{CPU}$ speed to take into consideration both $\mathrm{CPU}$ bound and I/O bound query processing, and study the impact of processor allocation and hash filtering in both cases. A detailed performance model on the cost of hash joins and system parameters used is given in Section 4 . In addition, we assume for simplicity that the values of attributes are uniformly distributed over all tuples in a relation and that the values of one attribute are independent of those in another. Thus, the cardinalities of resulting relations of joins can be estimated according to the formula used in prior work [6]. In the presence of data skew [37], we only have to modify the corresponding formula accordingly [10].

\subsection{The Effect of Hash Filters}

A hash filter (HF) built by relation $R_{i}$ on its attribute $A$, denoted by $H F_{R_{i}}(A)$, is an array of bits which are initialized to

0 s. Let $R_{i}(A)$ be the set of distinct values of attribute $A$ in $R_{i}$, and $h$ be the corresponding hash function employed. The $k$ th bit of $\operatorname{HF}_{R_{i}}(A)$ is set to one if there exists an $a \in R_{i}(A)$ such that $h(a)=k$. Similar to the effect of semijoins, it can be seen that before joining $R_{i}$ and $R_{j}$ on their common attribute $A$, probing the tuples of $R_{j}$ against $H F_{R_{i}}(A)$ and removing nonmatching tuples will reduce the number of tuples of $R_{j}$ to participate in the join. The join cost is thus reduced. An illustrative example of the use of hash filters can be found in Fig. 3, where an $H F_{R_{1}}(B)$ is built by $R_{1}$ and applied to $R_{2}$, with the corresponding hash function $h\left(b_{i}\right)=i \bmod 5$. It can be verified that after the application of $H F_{R_{1}}(B), R_{2}$ is reduced to the one given in Fig. $3 \mathrm{~b}$, thus reducing the join cost of $R_{1} \triangleright \triangleleft R_{2}$. In this paper, we shall develop an efficient scheme to interleave a bushy execution tree with hash filters

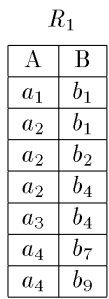

(a)

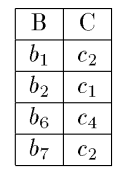

$R_{2}$ after the application of $H F_{R_{1}}(B) \rightarrow R_{2}$.

(b)

Fig. 3. An example of the use of hash filters.

to minimize the query execution cost for hash joins. Let $H F_{R_{i}}(A) \rightarrow R_{j}$ denote the application of a hash filter generated by $R_{i}$ on attributed $A$ to $R_{j}$. Note that the reduction of $R_{j}$ by $H F_{R_{i}}(A) \rightarrow R_{j}$ is proportional to the reduction of $R_{j}(A)$. The estimation on the size of the relation reduced is thus similar to estimating the reduction of projection on the corresponding attribute. Let $\rho_{i, A}$ be the reduction ratio by the application of $H F_{R_{i}}(A)$, and the cardinality of $R_{j}$ after $H F_{R_{i}}(A) \rightarrow R_{j}$ can be estimated as $\rho_{i, A}\left|R_{j}\right|$. Clearly, the determination of $\rho_{i, A}$ depends on the size of a hash filter since, as shown in Fig. 3, different attribute values may be hashed into a same hash entry.

As pointed out in [4], hashing $k=\left|R_{i}(A)\right|$ different values into a hash filter of $m$ bits is similar to the experiment of drawing $k$ balls from $m$ different balls with replacement. The following proposition, which is used to determine the effect of hash filters in our simulation, thus follows.

PROPOSITION 2 [4]. The reduction ratio by the application of $H F_{R_{i}}(A), \rho_{i, A}$ can be formulated as

$$
\rho_{i, A}= \begin{cases}1-\left(1-\frac{1}{m}\right)^{\left|R_{i}(A)\right|}, & \text { for } m<|A|, \\ \frac{\left|R_{i}(A)\right|}{|A|}, & \text { for } m \geq|A|,\end{cases}
$$

where $R_{i}(A)$ is the set of distinct values of attribute $A$ in $R_{i}$, and $m$ is the number of hash entries in a hash filter.

\section{Parallel Execution for Pipelined Hash JOINS}

In this section, we shall first derive a processor allocation scheme and then introduce methods to generate hash filters for a bushy execution tree.

\subsection{Processor Allocation for Bushy Trees Using Hash Joins}

As mentioned earlier, to exploit the opportunity of pipelining for hash join execution, one has to identify and execute a sequence of hash joins in the bushy tree in a way of 
pipelining. However, such regrouping of joins makes the execution dependency in the bushy tree intractable. Consequently, we shall first transform a bushy execution tree to an allocation tree where each node denotes a pipeline. Then, using the concept of synchronous execution time [6], processors are allocated to the nodes in the allocation tree. Explicitly, to minimize idleness of processors, the idea of synchronous execution time is to allocate processors for join execution judiciously so that inner relations in a pipeline can be made available at approximately the same time.

To transform a bushy tree to an allocation tree, we first identify the groups of joins in the bushy tree that could be pipelined. Then, an allocation tree can be obtained from the original bushy tree by merging each group of joins together. For example, suppose we determine seven groups of joins to be pipelined as shown in Fig. 4. By merging each pipeline in Fig. 4 into a single node, we obtain the corresponding allocation tree as given in Fig. 5. Next, we determine the number of processors allocated to each node (pipeline) in the allocation tree in the manner of top down. Clearly, all processors are allocated to the pipeline associated with the root in the allocation tree, say $S_{1}$ in Fig. 5, since it is the last pipeline to be performed. Those processors allocated to the pipeline on the root are then partitioned into several clusters which are assigned to execute the pipelines associated with the child nodes of the root in the allocation tree in such a way that those pipelines can be completed at approximately the same time. In other words, processors are so allocated that input relations for the root pipeline, say $S_{1}$ in Fig. 5, can be available about the same time to facilitate the execution of $S_{1}$. The above step for partitioning the processors for the root is then applied to all internal nodes in the allocation tree in a top down manner until each pipeline is assigned with a number of processors. Specifically, define the cumulative execution costs of a node in the allocation tree as the sum of the execution costs of all pipelines in the subtree under that internal node. Let $S_{i}$ be a pipeline associated with a node in the allocation tree $T_{A}$, and $C\left(S_{i}\right)$ be the set of child nodes of $S_{i}$ in $T_{A}$. Denote the cost of executing $S_{i}$ as $W\left(S_{i}\right)$. Then, the cumulative execution cost of the node with $S_{i}$, denoted by $C W\left(S_{i}\right)$, is determined by,

$$
C W\left(S_{i}\right)=W\left(S_{i}\right)+\sum_{S_{j} \in C\left(S_{i}\right)} C W\left(S_{j}\right) .
$$

Note that the cumulative execution cost of each node can be determined when the original bushy tree is built bottom up. Then, it is important to see that to achieve the synchronous execution time, when partitioning the processors of a node into clusters for its child nodes, one has to take into account the "cumulative execution costs" of the child nodes, rather than the "execution costs" of the pipelines associated with the child nodes. Denote the set of processors allocated to perform the pipeline $S_{x}$ as $P\left(S_{x}\right)$, and use \#P(S $\left.S_{x}\right)$ to represent the number of processors in $P\left(S_{x}\right)$. With $S_{x}$ being a child node of $S_{i}$, we have,

$$
\# P\left(S_{x}\right)=\left\lceil \# P\left(S_{i}\right) \frac{C W\left(S_{x}\right)}{\sum_{S_{j} \in C\left(S_{i}\right)} C W\left(S_{j}\right)}\right\rceil .
$$

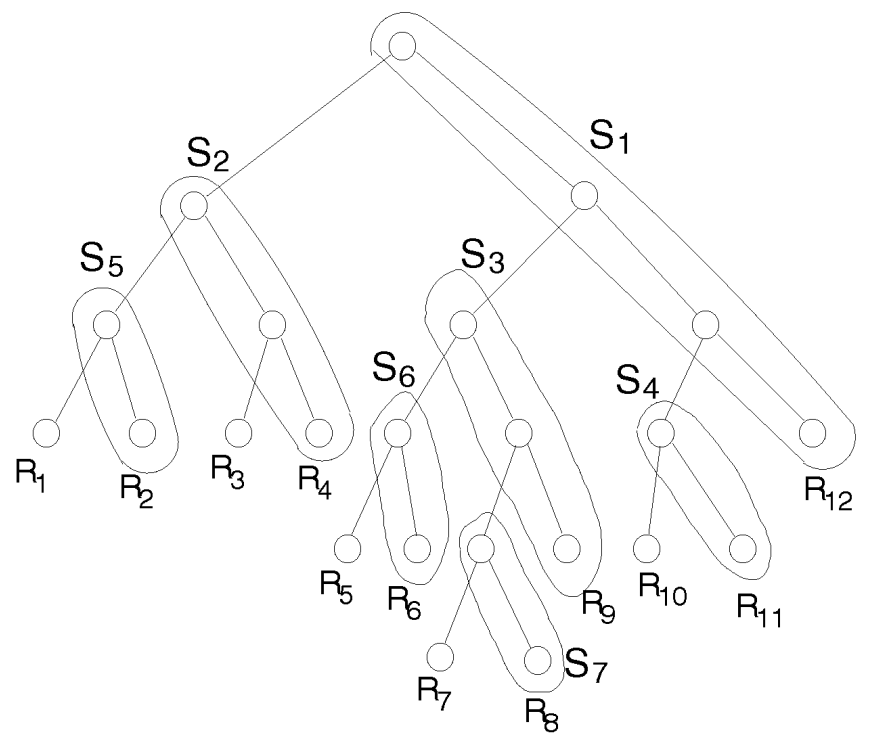

Fig. 4. Identifying pipelines in a bushy tree.

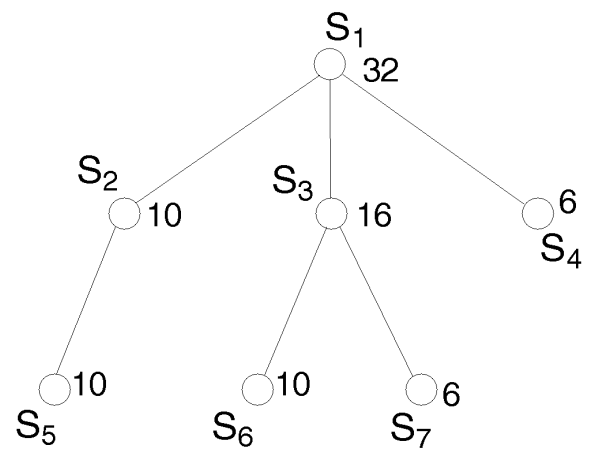

Fig. 5. Illustration of an allocation tree.

Formally, the processor allocation scheme based on an allocation tree can be described below.

Algorithm G: Allocating processors to a bushy tree utilizing pipelined hash joins.

Step 1: A join sequence heuristic is applied to determine a bushy execution tree $\mathrm{T}$.

Step 2: From the given bushy tree $\mathrm{T}$, determine the corresponding allocation tree $T_{A}$ by merging relations in each pipeline together.

Step 3: Based on (2), determine the cumulative workload of each node in $T_{A}$ in a bottom up manner.

Step 4: Using (3), allocate processors to each node in $T_{A}$ in a top down manner.

For illustrative purposes, given a total of 32 processors, a possible processor allocation for the allocation tree in Fig. 5 is shown by a set of numbers labeled next to nodes in the tree. As mentioned earlier, these numbers are so determined that all child nodes (pipelines) of each internal node can be completed approximately the same time. An example scenario for the execution of the bushy tree in Fig. 4 is given in Fig. 6, where the shaded area corresponds to the idleness of processors. It is worth mentioning that when the 


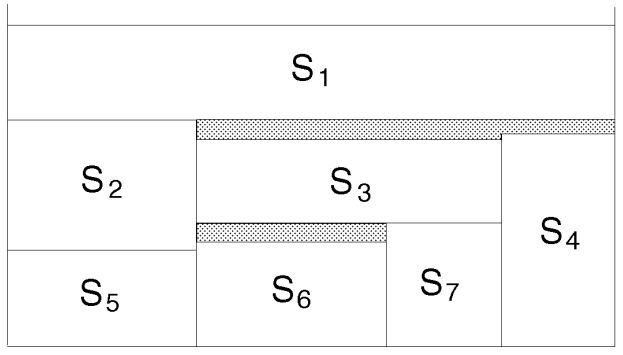

Fig. 6. Execution for the allocation tree in Fig. 5, where $X$ axis represents the number of processors and $Y$ axis corresponds to execution time.

number of processors passed to an internal node in a lower level of the tree is too few to be further partitioned for efficient execution of pipelines, sequential execution for the pipelines in its child nodes could be employed for a better performance. Note that the method described above determines the interpipeline processor allocation to execute pipelined hash joins. Clearly, how to distribute processors among many stages within a pipeline is also an important issue, whose discussion is beyond the scope of this paper. Readers interested in processor allocation within a pipeline are referred to [19].

\subsection{Interleaving a Bushy Execution Tree with Hash Filters}

To further improve the parallel execution of hash joins, we would like to employ hash filters to reduce the cost of each individual join. Note that, since the content of each relation may change after every database update, precomputed hash filters may become obsolete before any real use. Also, hash filters of interest depend on the joins to perform. We thus produce hash filters right before their use. Also, a hash filter has to be received in time for its processing, which is, however, difficult to achieve due to the nature of parallel execution of hash joins. It can be seen that, based on the processor allocation in algorithm $\mathrm{G}$, we have $S_{j} \in C\left(S_{i}\right) \Rightarrow$ $P\left(S_{j}\right) \subseteq P\left(S_{i}\right)$, meaning that a pipeline in a higher level of the allocation tree will be executed by those processors allocated to its offspring. This feature indeed resolves the timing constraint described above, since under such processor allocation, a hash filter received late can still be applied to a later pipeline which is executed by the same cluster of processors, thus avoiding incurring any transmission of hash filters among processors. Obviously, there are many methods conceivable to generate hash filters. For example, a straightforward method is to generate and apply hash filters to the bushy tree first and then to proceed with the normal execution. Such a scheme is termed "early generation" (denoted by EG) in what follows, whose algorithmic form can be described below.

Algorithm EG: Interleaving a bushy tree $T$ with hash filters. HF Sender: for each leaf node $R_{i}$ in T
${ }^{*}$ Let $R_{i}$ be a relation in pipeline $S_{j}$. ${ }^{*}$ begin
Let $J_{\text {att }}$ be the set of all join attributes in $R_{i}$. if $\left(J_{\text {att }} \neq \phi\right)$
begin

\author{
Scan $R_{i}$, and $\forall A \in J_{\text {att }}$, build $H_{R_{i}}(A)$ by $P\left(S_{j}\right)$. \\ Send $H_{R_{i}}(A)$ to $P\left(S_{k}\right)$, where $S_{k}$ contains a \\ relation joining with $R_{i}$ on $A$. end
end
HF Receiver: for each leaf node $R_{i}$ in $T$
begin \\ if $\left(R_{i}\right.$ receives all HFs for its join attributes) then \\ $R_{i}$ applies HFs to filter out nonmatching \\ tuples. \\ if ( $R_{i}$ is an inner relation) then building \\ the hash table \\ else starting tuple probing when inner \\ relations are available.
}

\section{end}

The first conditional statement in HF Sender to set up $J_{\text {att }}$ assures that only necessary hash filters will be generated and applied to other relations. Also, it can be seen that a relation will be scanned at most once to build HFs for attributes in $J_{\text {att }}$. Every relation, after receiving and utilizing all its filters, starts its normal operations. Clearly, depending on the cost and the benefit of hash filters, there can be many schemes to determine the hash filter generation. To provide more insights into the approach of hash filtering, extensive simulation will be conducted in Section 4 to evaluate various schemes using hash filters. Owing to the nature of hash joins, instead of being generated in advance, hash filters can be built together with the hash join operations to reduce the overhead associated. Specifically, hash filters from inner relations are built in their table-building phases and those from outer relations are built in their tuple-probing phases. Such an approach will be referred to as scheme CG, where CG stands for "complete generation." Also, as will be evaluated in Section 4, hash filters can be generated from inner relations only to reduce the cost of hash filter generation while attaining the desired reduction effect. This alternative is denoted by IG, standing for "inner relation generation." The conventional approach without using hash filters, denoted by NF (i.e., "no hash filters"), will also be implemented for a comparison purpose.

\section{Performance Study}

In this section, we describe the simulation model and results of experiments that we have conducted to study the relative performance of various processor allocation and hash filtering (HF) schemes described in Section 3. The architecture assumed in this study is a multiprocessor system with distributed memories and shared disks containing database data. This study also assumed that a processor activates one I/O process for every relation scan to read its portion of the relation from disk. Our goal was to evaluate the performance of processor allocation and hash filtering schemes in a variety of complex query environments. In the following, the performance model used to evaluate the benefit of different processor allocation and hash filtering schemes is first described in Section 4.1. Parameters used in the experiments are then given in Section 4.2. Finally, simulation results are presented and analyzed in Section 4.3. 


\subsection{Model Overview}

The performance model consists of three major components: Request Generator, Compiler, and Executor. Request Generator is responsible for generating query requests as follows. The number of relations in a query is determined by an input parameter, sn. Relation cardinalities and join attribute cardinalities are determined by a set of parameters: $R_{\text {card }}$, carv, $f_{d}(R), A_{\text {card }}$, attv, and $f_{d}(A)$. Relation cardinalities in a query are computed from a distribution function, $f_{d}(R)$, with a mean, $R_{\text {card }}$, and a deviation, carv. Cardinalities of join attributes are determined similarly by $A_{\text {card }}$, attv, and $f_{d}(A)$. There is a predetermined probability, $p$, that an edge (i.e., a join operation) exists between any two relations in a given query graph. The larger $p$ is, the larger the number of joins in a query will be. Note that some queries so generated may have disconnected query graphs. Without loss of generality, only queries with connected query graphs were used in our study, and those with disconnected graphs were discarded. Compiler takes a query request from Request Generator and produces a query plan in the form of a bushy tree. The bushy plan tree is determined by the minimum cost heuristic described in [6] that tries to perform the join with the minimal cost first.

Executor traverses the query plan tree and carries out join operations in parallel according to join sequence determined by the Compiler. Depending upon the hash filtering schemes simulated, hash filters of join attributes are generated at different stages of query execution. Hash filters are always generated from base relations, but they can be applied to base relations as well as to intermediate relations. After being generated, a hash filter is sent to all nodes (processors) that will perform join operation on the pipeline containing corresponding relation. For every hash filter received, it is applied to the corresponding base relation if the filter is received before the start of the relation scan. Otherwise, a received filter may be applied to the intermediate relation generated at the end of a pipelined join operation. In the worst case, a hash filter may not be received in time for applying to either base or intermediate relation. In this case, the hash filter will be discarded (is wasted).

In pipelined hash join operation, a tuple from outer relation can probe successively hash tables of multiple inner join relations in the same pipeline and a pipelined join operation will not start until all inner relations of the pipeline have completed building hash tables in memory. To minimize processor idle time and thus maximize the performance benefit of parallel execution of a query, processors should be allocated to join nodes such that the tablebuilding phase of all inner relations of a pipeline are completed approximately at the same time. To achieve this, the number of processors allocated to a join node is determined in a top down manner and the processors assigned to the parent node are divided among the child nodes according to the cumulative execution costs of the child nodes. In our simulation model, processor allocation is done as part of query compilation to simplify coding of the simulation model. Therefore, a query plan node in our experiment also contains the number of processors allocated to execute the node, in addition to the information about join relations, join attribute, join method, etc. In practice, allocation of processors to a join node can be deferred to runtime to provide more flexibility.

Two schemes for assigning processors to join nodes are studied in this paper. With the first scheme, the cumulative execution cost is computed for every node of the query plan tree generated by the Compiler. Processors are then allocated to execute a query plan node according to the cumulative execution cost of the node. In this scheme, the cumulative execution cost of a node is computed as the sum of the execution cost of joining the relations associated with its two child nodes plus the cumulative execution costs of its two child nodes. The cumulative execution cost of a leaf node is defined to be the cost of scanning the base relation. Henceforth, we shall refer to this as BOT (standing for Based on the Original Tree) processor allocation scheme. With the second scheme, a query plan tree is first transformed to a new tree, termed allocation tree, by lumping (join) nodes within each pipeline together to form a node of the new tree. Cumulative workload is then computed for each node of the allocation tree and processors are allocated to nodes based on the allocation tree (henceforth referred to as BAT scheme).

Our model computes both CPU and I/O costs of executing a query. Without loss of generality, we assume that each node (processor) has large enough physical memory to hold hash tables of all inner relations of a pipeline in memory at the same time (i.e., bucket overflow will not occur). But, the intermediate relation generated by a completed pipelined join operation is assumed to always be written to disks. When an intermediate relation is to be used by a later (pipelined) join operation, it is read from the disks and, at the same time, the hash table corresponding to the next join attribute is built in memory.

The number of CPU instructions executed to read and write a page of data from and to disk is assumed to be $I_{\text {read }}$ and $I_{\text {write }}$, respectively. The cost of extracting a tuple from a page in memory is assumed to be $I_{\text {tuple }}$ while the cost of building and probing hash tables is determined by the total number of tuples processed multiplied by the number of CPU instructions per tuple needed for table-building $\left(I_{\text {build }}\right)$ and tuple-probing $\left(I_{\text {probe }}\right)$. The total CPU cost of building a hash table in memory for a relation of $N$ tuples, including the cost of reading the relation from disks and that of extracting tuples from pages, is thus equal to $I_{\text {read }} \times N / p_{\text {size }}+$ $I_{\text {tuple }} \times N+I_{\text {build }} \times N$, where $p_{\text {size }}$ is the number of tuples per page. The CPU cost of carrying out the tuple-probing phase of a join operation, assuming the outer relation size of $N_{p}$ tuples, is equal to $I_{\text {probe }} \times N_{p}$, which is independent of the inner relation size $N$. To execute a pipeline of $n$ joins, the total CPU cost is then equal to

$$
\begin{aligned}
& \sum_{i=0}^{n} N_{i} / p_{\text {size }} \times I_{\text {read }}+\sum_{i=0}^{n} N_{i} \times I_{\text {tuple }}+ \\
& \sum_{i=1}^{n} N_{i} \times I_{\text {build }}+\sum_{i=1}^{n} N_{p_{i}} \times I_{\text {probe }}
\end{aligned}
$$

where $N_{i}$ is the inner relation size of $i$ th join (except $N_{0}$ that is the size of the outer relation of the first join operation) and $N_{p_{i}}$ denotes the outer/probing relation size of the $i$ th 
join operation in the pipeline. When $i=1, N_{p_{i}}$ is equal to $N_{0}$. For $i>1, N_{p_{i}}$ is the size of the result relation generated by the $(i-1)$ th join of the pipeline. The above cost does not include the spooling of intermediate relation (result) to disks at the end of a pipelined join. The cost of spooling is equal to $N_{p_{n+1}} / p_{\text {size }} \times I_{\text {write }}$ instructions, which are added to the CPU cost listed above for all pipelined join operations except the one that the head of the pipeline is the same as the root of the query plan tree.

The CPU processing time for executing a query is obtained by dividing the total number of CPU instructions per query by the CPU speed, $P_{\text {speed }}$. By separating the pathlength per query and the CPU speed, we have the flexibility of varying the CPU speed to make a query execution either $\mathrm{CPU}$ bound or I/O bound, and studying the impact of using hash filters in both cases.

$\mathrm{I} / \mathrm{O}$ cost for reading (writing) a relation of $N$ tuples is determined by disk service time per page, $t_{\text {pio }}$, multiplied by the total number of page read (written). To execute a pipeline of $n$ joins, $n+1$ relations are read from disks and the total $\mathrm{I} / \mathrm{O}$ cost for reading relations is thus equal to

$$
\sum_{i=0}^{n} N_{i} / p_{\text {size }} \times t_{\text {pio }} \text {. }
$$

The intermediate relation generated by a pipelined join is always spooled to disks except the one that is generated by the pipeline containing the root of the query plan tree. This increases the I/O cost by an amount of $N_{p_{n+1}} / p_{\text {size }} \times t_{\text {pio }}$. As before, $N_{p_{n+1}}$ denotes the size of the result relation generated by the $n$th (last) join of the pipeline.

For schemes that generate and apply hash filters, the CPU cost of generating a hash filter from a relation of size $N$ is computed by multiplying $I_{\text {hash }}$ by $N$, while the cost of applying a hash filter to a relation of size $N$ is computed by multiplying $I_{\text {apply }}$ by $N$. Where $I_{\text {hash }}$ is the number of CPU instructions required to generate a hash value from an input tuple and set the corresponding bit in the hash filter, and $I_{\text {apply }}$ is the number of instructions needed to check whether an attribute value of a tuple has a match in the filter, and, if that bit is set, it adds the tuple to a temporary relation to be joined later. Note that hash filter generation phase can be combined with the base relation scan for join operation, thus avoiding I/O overhead in hash filter generation (the IG and CG schemes). On the other hand, if all hash filters are generated in a separated phase, prior to the start of the first join operation of the query (the EG scheme), $\mathrm{N} / \mathrm{p}_{\text {size }}$ additional I/Os per relation are required. Also note that, in our simulation model, hash filters are implemented as bit-vectors and can, in general, fit in memory, thus minimizing extra I/Os required for maintaining them.

\subsection{Parameter Setting}

We select queries of five sizes, i.e., queries with four, eight, 12,16 , and 20 relations. This set of selections covers a wide spectrum of query sizes ranging from a simple three-way join to a more than 20-way join. For each query size, 500 query graphs were generated, and, as mentioned in Section 4.1 , only queries with connected query graphs are used in our study.

To conduct the simulation, [2], [8], [15], and [28] were referenced to determine the values of simulation parameters. Table 1 summarizes the parameter settings used in the simulation. The number of processors in the system is set to 32 and the speed of the processor is assumed to be three MIPS. The number of CPU instructions for reading $\left(I_{\text {read }}\right)$ and writing $\left(I_{\text {write }}\right)$ a page is set to 5,000 , while those for extracting a tuple from page in memory is set to 300. Applying hash function to an attribute to build either hash table or hash filter is assumed to take 100 instructions each, while probing hash table or probing hash filter to filter out nonmatching tuples is assumed to consume 200 instructions each. Each page is assumed to contain 40 tuples and disk service time per page (both read and write) is assumed to be 20 milliseconds. The size of a relation varies from $700 \mathrm{~K}$ to $1,300 \mathrm{~K}$ tuples, while each attribute has from $600 \mathrm{~K}$ to $1,000 \mathrm{~K}$ distinct values. Finally, the distribution functions for relation cardinality and attribute cardinality are both assumed to be uniform.

TABLE 1

PARAMETERS USED IN SIMULATION

\begin{tabular}{||l|l|l||}
\hline parameters & meaning & setting \\
\hline \hline$n_{p}$ & num. of processors & 32 \\
\hline$P_{\text {speed }}$ & processing speed & $3 \mathrm{MIPS}$ \\
\hline$I_{\text {read }}$ & inst. to read a page from disk & 5,000 \\
\hline$I_{\text {write }}$ & inst. to write a page to disk & 5,000 \\
\hline$I_{\text {tuple }}$ & inst. to get a tuple from mem. & 300 \\
\hline$I_{\text {build }}$ & inst./tuple for table-building & 100 \\
\hline$I_{\text {prob }}$ & inst./tuple for tuple-probing & 200 \\
\hline$I_{\text {hash }}$ & inst./tuple to get hash value & 100 \\
\hline$I_{\text {apply }}$ & inst. to process a hashed tuple & 200 \\
\hline$P_{\text {size }}$ & tuples per page & 40 tuples \\
\hline$t_{\text {pio }}$ & disk service time per page & $20 \mathrm{~ms}$ \\
\hline$R_{\text {card }}$ & mean of relation card. & $1 \mathrm{M} \mathrm{tuples}$ \\
\hline$A_{\text {card }}$ & mean of attr. card. & $800 \mathrm{~K}$ \\
\hline carv & deviation of relation card. & $\pm 100 \mathrm{~K}-300 \mathrm{~K}$ \\
\hline attv & deviation of attr. card. & $\pm 100 \mathrm{~K}-200 \mathrm{~K}$ \\
\hline$f_{d}(A)$ & dist. function of attr. card. & uniform \\
\hline$f_{d}(R)$ & dist. function of relation card. & uniform \\
\hline
\end{tabular}

\subsection{Simulation Results}

In the simulation program, which was coded in C, the action for each individual relation to go through join operation, as well as hash filter generation and application, was simulated. For each query in the simulation, four hash filtering schemes, i.e., NF (no filter), EG (early generation), IG (inner generation), and CG (complete generation), were applied to execute the query, and the CPU cost, I/O cost as well as total response time for each scheme was obtained.

The experiment was carried out in two stages. In the first stage, we studied the relative performance of different processor allocation schemes. Specifically, we wanted to 
explore the benefit, in terms of reduction in query response time, of allocating processors to join nodes based on allocation tree (BAT) in a multiprocessor database environment. In the second stage, we fixed the processor allocation scheme to BAT and ran a set of experiments to study the relative performance of the four different hash filtering schemes.

\subsubsection{Alternative Processor Allocation Schemes}

\section{Experiment 1: Performance of Processor Allocation Schemes}

In the first experiment, both attv and carv were set to $100 \mathrm{~K}$ and the number of processors was set to 32 . The number of processors allocated to execute a join node is determined by either BOT or BAT. Fig. 7 shows the average response time of the queries for the NF method using either BOT or BAT processor allocation schemes. We deliberately turned off hash filter application in this set of experiments to demonstrate the importance of processor allocation in multiprocessor database systems. In this figure, the ordinate is the response time in milliseconds, while the abscissa denotes the number of relations in a query. As illustrated in Fig. 7, response time with BAT is significantly lower than that with BOT in all queries evaluated. Using BOT processor allocation scheme, average response time for a join query involving four relations $(\mathrm{sn}=4)$ is about 354 seconds, while it is 3,874 seconds for the case of $s n=20$. When a query plan is first transformed to an allocation tree and processors are allocated to join nodes based on the allocation tree, the average response times for $s n=4$ and $s n=20$ were cut to 147 seconds and 1,235 seconds, respectively. This is an improvement of more than 50 percent for query with sn $=4$ and improvement of about 70 percent for $\mathrm{sn}=20$.

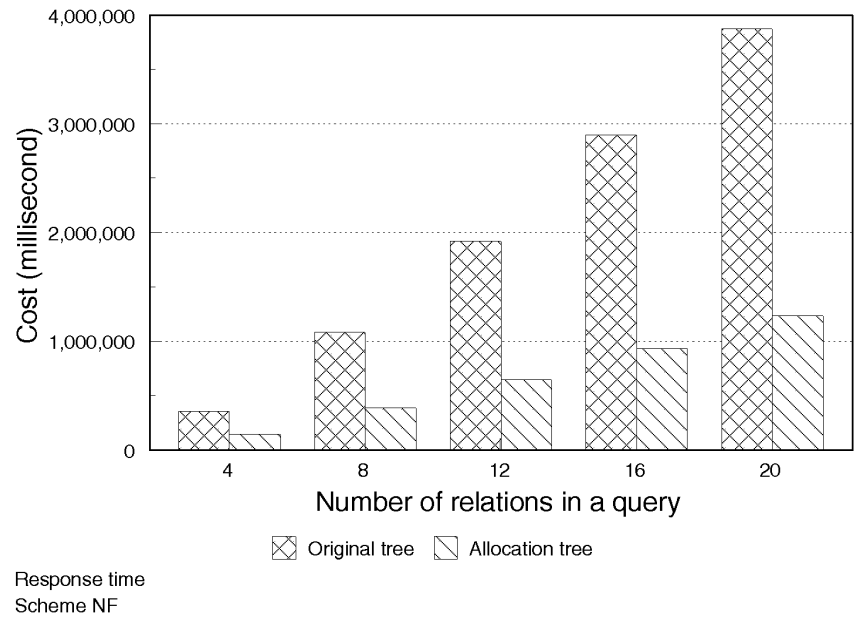

Fig. 7. The response time for the NF scheme.

This experiment shows that the BOT processor allocation scheme, which was demonstrated to provide superior performance with sort-merge and nested-loop join methods [6], does not perform well with pipelined hash join method. This is because, with pipelined hash join, a join operation does not start until hash tables of all inner relations of the pipeline are materialized in memory. Therefore, it is best to allocate processors to join nodes such that all inner relations are materialized around the same time (and thus minimize processor idle time). The BAT processor allocation scheme takes this into consideration and assigns processors to join nodes accordingly. As a result, the BAT scheme performs significantly better than the BOT scheme in our experiments.

\section{Experiment 2: Impact on HF Schemes}

In this experiment, we fixed the number of relations in a query to $12(\mathrm{sn}=12)$ and ran one simulation for each $\mathrm{HF}$ scheme described in Section 3. carv and attv were again set to $100 \mathrm{~K}$ each and the number of processors in the system was set to 32. This experiment was intended to study how each HF scheme performs under different processor allocation schemes. Fig. 8 shows the results of this experiment. With BAT processor allocation scheme, the response time of the query is significantly lower than that with BOT across all HF schemes. Specifically, the response time is reduced by about 66 percent with NF and EG and by about 69 percent with IG and CG when the processor allocation scheme is changed from BOT to BAT. Fig. 8 also shows that, with both allocation schemes, IG and CG perform comparably well and both perform better than EG and NF. EG produces moderately higher response time than either IG or CG, while NF has the worst response time.

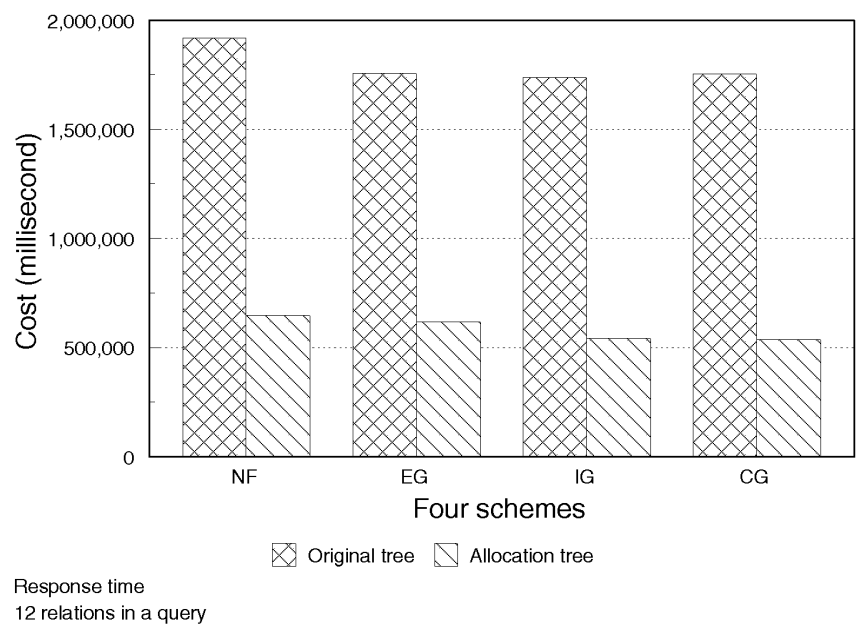

Fig. 8. The response time of each scheme with 12 relations (NF: no filter, EG: early generation, IG: inner generation, CG: complete generation).

Table 2 shows the breakdown of the response time of the query for each of the four HF schemes. The I/O cost and CPU cost columns are, respectively, the average $\mathrm{I} / \mathrm{O}$ time and CPU time consumed by the query, while the fourth column is the time that processors are idle, waiting for peers to complete preceding join operations. The \%-of-RPT column shows the fraction of total response time that is due to processor waiting for peers to finish and thus idle. For comparison purpose, the table includes in the last column the percentage of response time that a processor is idle when BOT is used. Table 2 indicates that, with BAT, processor synchronization (waiting time) contributes (on average) less than 12 percent to the total response time, while it contributes more than 70 percent in the case of BOT. 
TABLE 2

BREAKDOWN OF RESPONSE TIME IN MILLISECOND-BAT

\begin{tabular}{||l|l|l|l|c|c||}
\hline schemes & I/O cost & CPU cost & idle time & \% of RPT & \% of RPT(BOT) \\
\hline \hline NF & 468,597 & 119,531 & 59,084 & $9.1 \%$ & $69 \%$ \\
\hline EG & 399,967 & 142,387 & 75,299 & $12.2 \%$ & $69 \%$ \\
\hline IG & 355,665 & 124,236 & 62,431 & $11.5 \%$ & $74 \%$ \\
\hline CG & 326,986 & 133,204 & 75,320 & $14.1 \%$ & $75 \%$ \\
\hline
\end{tabular}

\subsubsection{Performance of HF Schemes}

In the second stage of our simulation study, processor allocation scheme was fixed to BAT (unless indicated otherwise). Our goal was to study the effectiveness of alternative $\mathrm{HF}$ schemes for queries with varying complexity. As described in Section 2, the EG scheme generates hash filters from all base relations before the start of actual join operation and then applies hash filters to base relations during join operations. This scheme provides the maximum reduction effect of hash filtering because all hash filters are available (and thus can be applied) before the start of any join operation. But, it also incurs the highest overhead because it needs to go through one extra round of disk I/O to read the base relations from disks in order to generate the hash filters. IG was designed to minimize the overhead associated with the hash filter generation while CG was designed to maximize the effects of hash filtering. With IG, hash filters are generated from inner base relation only and they are generated at the time when inner relations are read from disks to build in memory hash tables. Consequently, no extra I/O are incurred and thus the overhead of applying hash filter is minimized. With CG, hash filters are also generated from all base relations. But unlike EG, CG generates hash filters from a relation when the relation is to be joined next and thus no extra I/Os are incurred. However, because hash filters from outer relations are not generated until the relations are to be joined next, they are generally not received in time for applications by partner relations. As a result, the number of hash filters generated with $C G$ is equivalent to that with EG, but the number of hash filters applied per query is significantly less with CG.

Experiment 3: Low Variance, carv $=100 \mathrm{~K}$, and attv $=100 \mathrm{~K}$ In this experiment, relation cardinality ranges from $900 \mathrm{~K}$ to $1,100 \mathrm{~K}$ tuples, while attribute cardinality ranges from $700 \mathrm{~K}$ to $900 \mathrm{~K}$. The average CPU cost, I/O cost, and response time for this experiment are shown in Figs. 9, 10, and 11, respectively, where AT stands for allocation tree. In these figures and all following figures, the ordinate is the response time in milliseconds, while the abscissa denotes the number of relations in a query. Figs. 9 and 10 show that with three MIPS CPU, these queries using the pipelined hash join method are $\mathrm{I} / \mathrm{O}$ bound. The $20 \mathrm{~ms}$ page I/O time setting assumes no prefetching or disk buffering (e.g., reading one track at a time). The experiment also assumes that one I/O process is activated on each processor for every relation scan. Note that this experiment could become CPU bound if disk buffering or parallel I/O strategy (activate multiple I/O processes per processor for every relation scan) was used.

Fig. 9 shows that applying hash filters results in a slightly higher CPU cost with the CG methods than with $\mathrm{NF}$, while it results in a significantly higher CPU cost with the EG method. IG consumes approximately the same amount of CPU resource as NF. This experiment shows that, with respect to CPU cost, the benefits of applying hash filter (size reduction) is overshadowed by the cost of generating and applying hash filters with both EG and CG across all queries evaluated. This is because, without bucket overflow, the CPU cost of a hash join is linearly proportional to the size of two input relations. In the simulation, we assume that intermediate relations are not spooled to disks except at the end of a pipelined join and that bucket overflow does not occur. Consequently, the benefit of reduction in intermediate relation size shows up only at the end of a pipelined join, resulting in lower benefit of applying hash filter. If more intermediate relations are spooled to disks, the benefit of applying hash filter will be much higher.

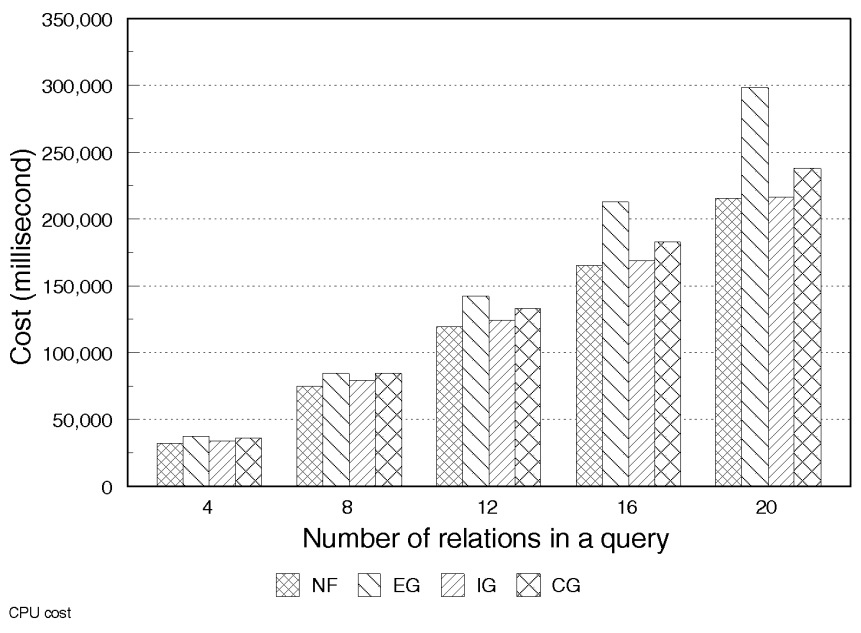

Fig. 9. The CPU cost for each scheme based on AT.

Fig. 10 shows that applying hash filters results in a slight performance improvement in $\mathrm{I} / \mathrm{O}$ cost when $s n$ is small $(s n \leq 8)$. The improvement increases significantly as the number of relations in a query increases. This is because the number of pipelines increases as $s n$ increases and, as explained in the foregoing paragraph, the benefit of size reduction thus becomes more prominent. It can also be seen from Fig. 10 that $C G$ performs the best among all schemes evaluated while NF is outperformed by all other schemes. As previously described, neither CG nor IG incurs any I/O overhead in generating and applying hash filters. Because more hash filters are generated and likely more hash filters are applied with CG, the size of intermediate relations, on average, is likely to be smaller with CG than with IG. Consequently, CG provides the lowest I/O cost among all schemes evaluated, while IG, the second lowest. For EG, it applies more hash filters than all other schemes and thus provides the maximum reduction effect. However, it scans 


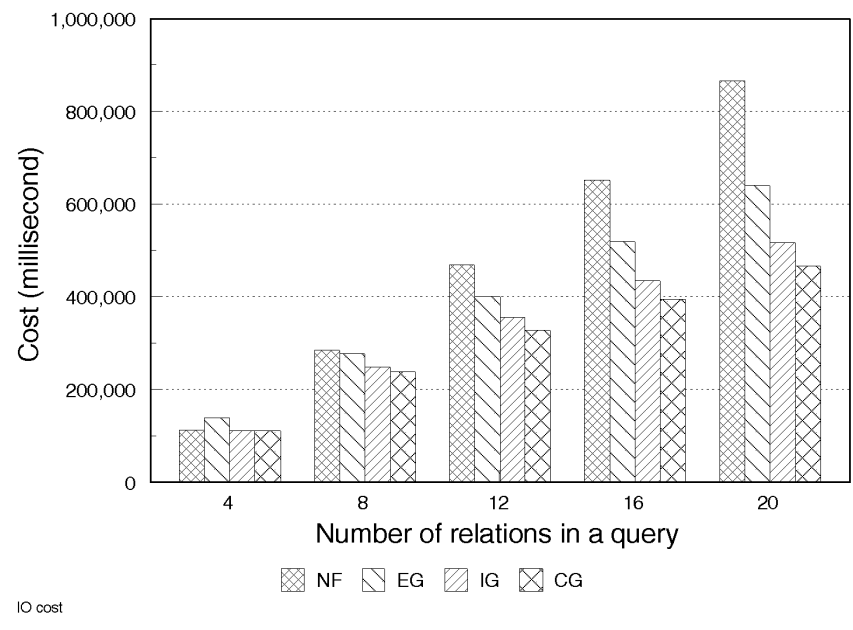

Fig. 10. The $\mathrm{l} / \mathrm{O}$ cost for each scheme based on AT.

the base relations one extra time to build the hash filters. As a result, the total $\mathrm{I} / \mathrm{O}$ cost with $\mathrm{EG}$ is lower than that with NF, but higher than either IG or CG.

Fig. 11 shows that, except in the case of $s n=4$, total query response time can always be reduced by applying hash filters. When $s n=4$, neither IG nor CG shows any performance improvement or performace degradation over NF. With EG, however, the response time increases by about 21 percent compared to that with NF. When $s n=20$, the response time is reduced, related to NF, by more than 20 percent with either IG or CG, while the improvement is about three percent with EG. The result clearly demonstrates that either IG or CG is an effective means for reducing response time of complex query execution using the pipelined hash join method, especially when $s n$ is large. The experiment also shows that the extra system resource consumed by EG outweighs the benefit of size reduction when $s n \leq 8$. When $s n$ is greater than eight, EG also shows performance improvement over NF, but the reduction in response time is limited to less than five percent.

For reference, Fig. 12 shows the response time of the queries for each HF schemes using the BOT processor allo-

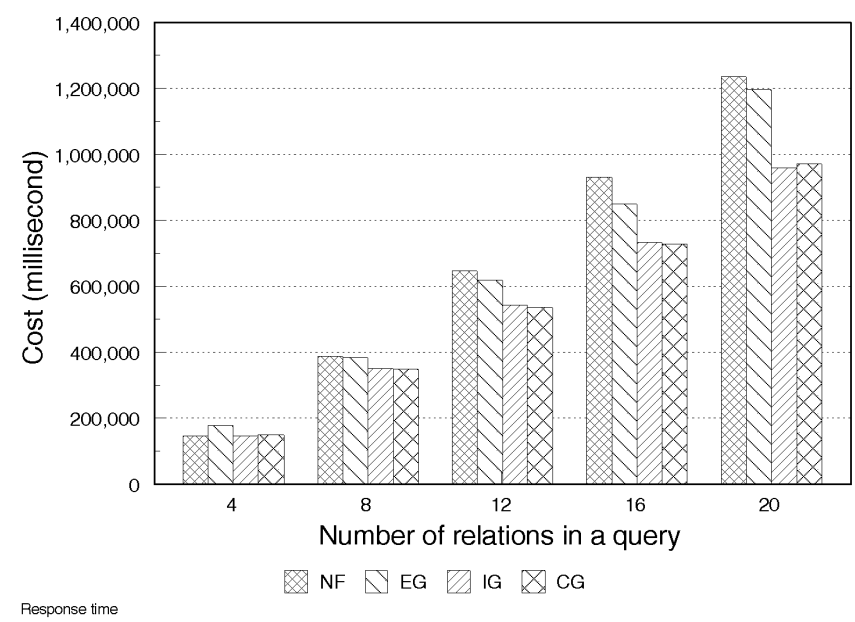

Fig. 11. The average response time for each scheme based on AT.

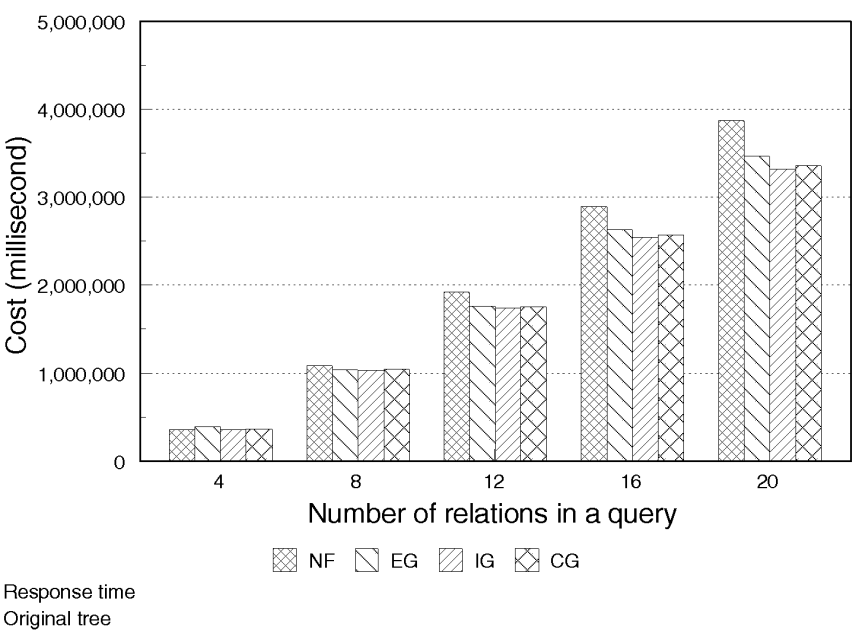

Fig. 12. The average response time for each scheme based on BOT processor allocation.

cation scheme. As indicated in the figure, IG remains to be the best scheme evaluated and the improvement over NF in response time is about 15 percent when $s n=20$. Compared to Fig. 11, Fig. 12 shows that the benefit of applying hash filter is lower with BOT. This is because, with BOT, processor idle time (waiting for peers to complete preceding join operations) contributes to a significant portion of the total response time and, as a result, the saving in join execution time by applying hash filter becomes less dominant.

Experiment 4: High Variance, $\operatorname{carv}=300 \mathrm{~K}$, and attv $=200 \mathrm{~K}$

In this experiment, we increased the variance of relation cardinality from $100 \mathrm{~K}$ to $300 \mathrm{~K}$ and the variance of attribute cardinality from $100 \mathrm{~K}$ to $200 \mathrm{~K}$. By changing the variances of relation and attribute cardinalities, the effectiveness of hash filters on hash based join operations with varied cardinalities can be studied. Figs. 13, 14, and 15 show, respectively, the CPU cost, the I/O cost, and the response time for each scheme. Similar to Fig. 9, Fig. 13 shows that EG consumes the most CPU resource, while NF and IG consume the least. On the other hand, Fig. 14 shows that, except when $s n=4$, NF consumes the most disk resource, while CG, the least. Overall, as indicated in Fig. 15, when $s n>4$, both IG and CG generate noticeable performance (response time) improvement over NF. When $s n=20$, applying hash filters can improve the response time by more than 20 percent with the IG scheme. Compared to the results in Experiment 3, these three figures indicate that the effectiveness of applying hash filter is very stable when the variances of relation cardinalities and attribute cardinalities increase. As before, this experiment shows that IG is the best scheme among all schemes evaluated, while CG is a close second.

\section{Conclusions}

In this paper, we explored two important issues, processor allocation and the use of hash filters, to improve the parallel execution of hash joins. To exploit the opportunity of pipelining for hash join execution, a scheme to transform a bushy execution tree to an allocation tree was first devised. Also, the approach of hash filtering has been investigated to 
further improve the parallel execution of hash joins. Extensive performance studies have been conducted to demonstrate the importance of processor allocation and to evaluate various schemes using hash filters via simulation.

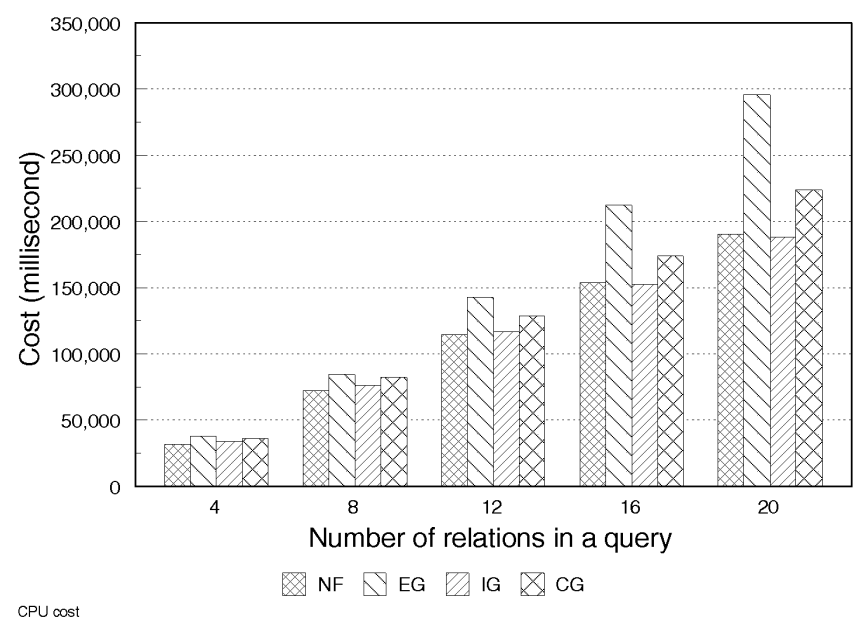

Fig. 13. The CPU cost for each scheme, high variance.

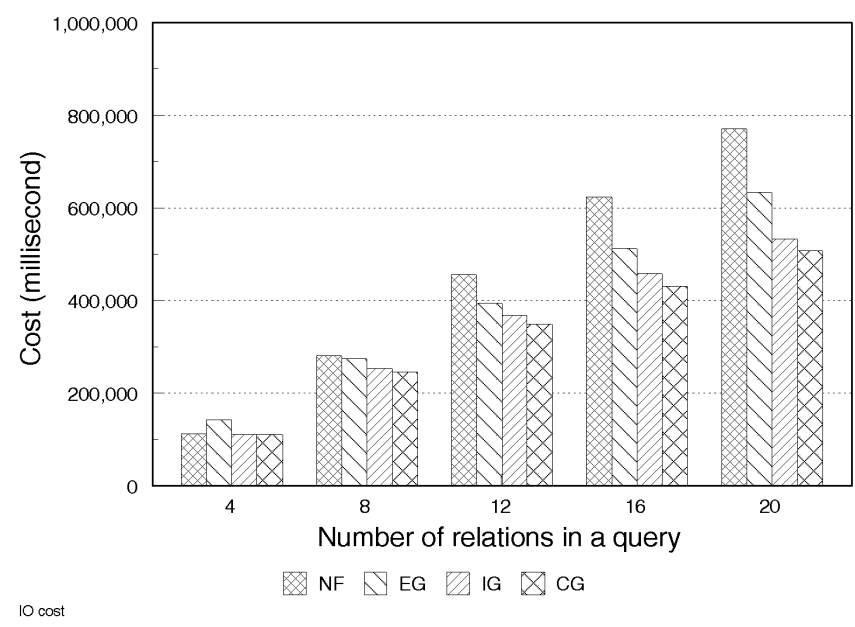

Fig. 14. The $\mathrm{I} / \mathrm{O}$ cost for each scheme, high variance.

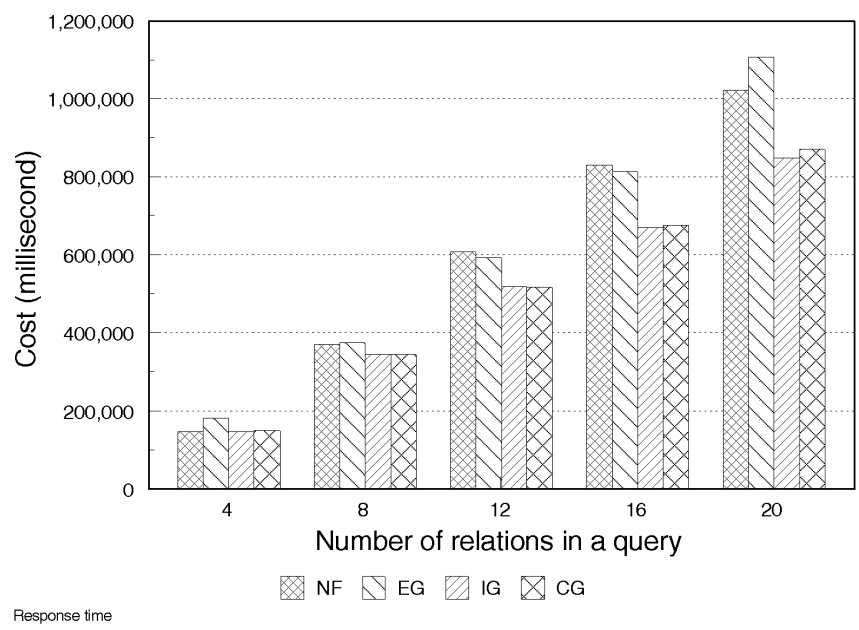

Fig. 15. The average response time for each scheme, high variance.
Among all schemes for hash filtering evaluated, the one to build hash filters for inner relations emerged as a winner. It was experimentally shown that processor allocation is, in general, the dominant factor to performance, and the effect of hash filtering becomes more prominent as the number of relations in a query increases. It is worth mentioning that a query optimizer, in general, realizes a query plan according to some estimations. If those estimations are later found to be very far from execution results, re-generation of query plans will be needed. How to determine a regeneration criterion is a problem of practical importance and a matter of our future study.

\section{ACKNOWLEDGMENTS}

Ming-Syan Chen is supported in part by the National Science Council, Project No. NSC 86-2621-E-002-023-T, Taiwan, Republic of China.

\section{REFERENCES}

[1] C. Baru et al., "An Overview of DB2 Parallel Edition," Proc. ACM SIGMOD, pp. 460-462, May 1995.

[2] D. Bitton and J. Gray, "Disk Shadowing," Proc. 14th Int'l Conf. Very Large Data Bases, 1988.

[3] H. Boral, W. Alexander et al., "Prototyping Bubba, A Highly Parallel Database System," IEEE Trans. Knowledge and Data Eng., vol. 2, no. 1, pp. 4-24, Mar. 1990.

[4] M.-S. Chen, H.-I. Hsiao, and P.S. Yu, "Applying Hash Filerts to Improving the Execution of Bushy Trees," Proc. 19th Int'l Conf. Very Large Data Bases, pp. 505-516, Aug. 1993.

[5] M.-S. Chen, M.-L. Lo, P.S. Yu, and H.C. Young, "Applying Segmented Right-Deep Trees to Pipelining Multiple Hash Joins," IEEE Trans. Knowledge and Data Eng., vol. 7, no. 4, pp. 656-668, Aug. 1995.

[6] M.-S. Chen, P.S. Yu, and K.L. Wu, "Scheduling and Processor Allocation for Parallel Execution of Multi-Join Queries," Proc. Eighth Int'l Conf. Data Eng., pp. 58-67, Feb. 1992.

[7] D.J. DeWitt and R. Gerber, "Multiprocessor Hash-Based Join Algorithms," Proc. 11th Int'l Conf. Very Large Data Bases, pp. 151-162, Aug. 1985.

[8] D.J. DeWitt, S. Ghandeharizadeh, D.A. Schneider, A. Bricker, H.I. Hsiao, and R. Rasmussen, "The Gamma Database Machine Project," IEEE Trans. Knowledge and Data Eng., vol. 2, no. 1, pp. 44-62, Mar. 1990.

[9] D.J. DeWitt and J. Gray, "Parallel Database Systems: The Future of High Performance Database Systems," Comm. ACM, vol. 35, no. 6, pp. 85-98, June 1992.

[10] D. Gardy and C. Puech, "On the Effect of Join Operations on Relation Sizes," ACM Trans. Database Systems, vol. 14, no. 4, pp. 574603, Dec. 1989.

[11] B. Gerber, "Informix Online XPS," Proc. ACM SIGMOD, p. 463, May 1995.

[12] R. Gerber, "Dataflow Query Processing Using Multiprocessor Hash-Partitioned Algorithms," Technical Report 672, Computer Science Dept., Univ. of Wisconsin-Madison, Oct. 1986.

[13] W. Hong, "Exploiting Inter-Operator Parallelism in XPRS," Proc. ACM SIGMOD, pp. 19-28, June 1992.

[14] W. Hong and M. Stonebraker, "Optimization of Parallel Query Exeuction Plans in CPRS," Proc. First Conf. Parallel and Distributed Information Systems, pp. 218-225, Dec. 1991.

[15] H.-I. Hsiao and D. DeWitt, "A Performance Study of Three High Availability Data Replication Strategies," Proc. First Conf. Parallel and Distributed Information Systems, pp. 79-84, Dec. 1991

[16] Y.E. Ioannidis and Y.C. Yang, "Left-Deep vs. Bush Trees: An Analysis of Strategy Spaces and Its Implication for Query Optimization," Proc. ACM SIGMOD, pp. 168-177, May 1991.

[17] M. Kitsuregawa, H. Tanaka, and T. Moto-Oka, "Architecture and Performance of Relational Algebra Machine GRACE," Proc. Int'l Conf. Parallel Processing, pp. 241-250, Aug. 1984. 
[18] R. Krishnamurthy, H. Boral, and C. Zaniolo, "Optimization of Nonrecursive Queries," Proc. 12th Int'l Conf. Very Large Data Bases, pp. 128-137, Aug. 1986.

[19] M.-L. Lo, M.-S. Chen, C.V. Ravishankar, and P.S. Yu, "On Optimal Processor Allocation to Support Pipelined Hash Joins," Proc. ACM SIGMOD, pp. 69-78, May 1993.

[20] R.A. Lorie, J.-J. Daudenarde, J.W. Stamos, and H.C. Young, "Exploiting Database Parallelism in a Message-Passing Multiprocessor," IBM J. Reserach and Development, vol. 35, nos. 5/6, pp. 681695, Sept./Nov. 1991.

[21] H. Lu, M.-C. Shan, and K.-L. Tan, "Optimization of Multi-Way Join Queries for Parallel Exeuction," Proc. 17th Int'l Conf. Very Large Data Bases, pp. 549-560, Sept. 1991.

[22] H. Lu, K.L. Tan, and M.-C. Shan, "Hash-Based Join Algorithms for Multiprocessor Computers with Shared Memory," Proc. 16th Int'l Conf. Very Large Data Bases, pp. 198-209, Aug. 1990.

[23] P. Mishra and M.H. Eich, "Join Processing in Relational Databases," ACM Computing Surveys, vol. 24, no. 1, pp. 63-113, Mar. 1992.

[24] E. Omiecinski and E.T. Lin, "The Adaptive-Hash Join Algorithm for a Hypercube Multicomputer," IEEE Trans. Parallel and Distributed Systems, vol. 3, no. 3, pp. 334-349, May 1992.

[25] H. Pirahesh, C. Mohan, J. Cheng, T.S. Liu, and P. Selinger, "Parallelism in Relational Data Base Systems: Architectural Issues and Design Approaches," Proc. Second Int'l Symp. Databases in Parallel and Distributed Systems, pp. 4-29, July 1990.

[26] N. Roussopoulos and H. Kang, "A Pipeline N-Way Join Algorithm Based on the 2-Way Semijoin Program," IEEE Trans. Knowledge and Data Eng., vol. 3, no. 4, pp. 461-473, Dec. 1991.

[27] D. Schneider, "Complex Query Processing in Multiprocessor Database Machines," Technical Report 965, Computer Science Dept., Univ. of Wisconsin-Madison, Sept. 1990.

[28] D. Schneider and D.J. DeWitt, "A Performance Evaluation of Four Parallel Join Algorithms in a Shared-Nothing Multiprocessor Environment," Proc. ACM SIGMOD, pp. 110-121, 1989.

[29] D. Schneider and D.J. DeWitt, "Tradeoffs in Processing Complex Join Queries via Hasing in Multiprocessor Database Machines," Proc. 16th Int'l Conf. Very Large Data Bases, pp. 469-480, Aug. 1990.

[30] P.G. Selinger, M.M. Astrahan, D.D. Chamberlin, R.A. Lorie, and T.G. Price, "Access Path Selection in a Relational Database Management System," Proc. ACM SIGMOD, pp. 23-34, 1979.

[31] E. Shekita, H.C. Young, and K. Tan, "Multi-Join Optimization for Symmetric Multiprocessors," Proc. 19th Int'l Conf. Very Large Data Bases, pp. 479-492, Aug. 1993.

[32] M. Stonebraker, R. Katz, D. Patterson, and J. Ousterhout, "The Design of XPRS," Proc. 14th Int'l Conf. Very Large Data Bases, pp. 318330, 1988.

[33] A. Swami, "Optimization of Large Join Queries: Combining Heuristics with Combinatorial Techniques," Proc. ACM SIGMOD, pp. 367376, 1989.

[34] A. Swami and A. Gupta, "Optimization of Large Join Queries," Proc. ACM SIGMOD, pp. 8-17, 1988

[35] A. Wilschut and P. Apers, "Dataflow Query Execution in Parallel Main-Memory Environment," Proc. First Conf. Parallel and Distributed Information Systems, pp. 68-77, Dec. 1991.

[36] A.N. Wilschut, J. Flokstra, and P.M.G. Apers, "Parallel Evaluation of Multi-Join Queries," Proc. ACM SIGMOD, pp. 115-126, May 1995.

[37] J.L. Wolf, P.S. Yu, J. Turek, and D.M. Dias, "A Parallel Hash Join Algorithm for Managing Data Skew," IEEE Trans. Parallel and Distributed Systems, vol. 4, no. 12, pp. 1,355-1,371, Dec. 1993.

[38] P.S. Yu, M.-S. Chen, H. Heiss, and S.H. Lee, "On Workload Characterization of Relational Database Environments," IEEE Trans. Software Eng., vol. 18, no. 4, pp. 347-355, Apr. 1992.

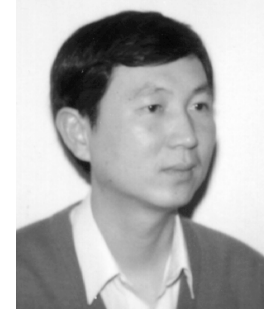

Hui-I Hsiao received a BS degree from National Taiwan University and MS and PhD degrees in computer science from the University of Wisconsin at Madison.

Dr. Hsiao joined the IBM T.J. Watson Reserach Center as a research staff member in September 1990. He is currently the manager of the Parallel Databases Project. Working with IBM Toronto, his group is responsible for research, design, and development of DB2 Parallel Edition-a highly scalable parallel database system on open system platform. Prior to joining IBM, he worked as a software engineer at Nicolet Instrument Corporation, where he received a Nicolet Associate Fellow award. His research interests include parallel database architecture, parallel query and transaction processing strategies, and database performance analysis.

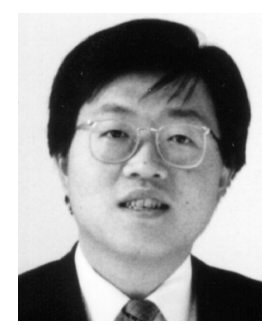

Ming-Syan Chen (S'87-M'88-SM'93) received the BS degree in electrical engineering from National Taiwan University, Taipei, Taiwan, in 1982, and the $\mathrm{MS}$ and $\mathrm{PhD}$ degrees in computer, information, and control engineering from the University of Michigan, Ann Arbor, in 1985 and 1988, respectively.

$\mathrm{Dr}$. Chen was a research staff member at the IBM T.J. Watson Research Center, Yorktown Heights, New York, from 1988 to 1996, primarily involved in projects related to parallel databases, multimedia systems, and internet applications. He is currently a faculty member of the Electrical Engineering Department, National Taiwan University, Taipei, Taiwan. He has published more than 75 refereed international journal/conference papers in the areas of parallel and distributed database systems, query processing, data mining, and multimedia systems.

Dr. Chen is currently serving on the editorial board of IEEE Transactions on Knowledge and Data Engineering. In addition to serving as a program committee member for many conferences, Dr. Chen was a tutorial speaker on parallel databases at the 11th IEEE International Conference on Data Engineering in 1995 and was a guest co-editor for IEEE Transactions on Knowledge and Data Engineering for a special issue on data mining in December 1996. He holds, or has applied for, 17 U.S. patents in the areas of interactive video playout, video server design, and concurrency and coherency control protocols. He received the Outstanding Innovation award from IBM Corporation in 1994 for his contribution to parallel transaction design and implementation for a major database product, and has received numerous awards for his inventions and patent applications.

Dr. Chen is a senior member of the IEEE and a member of the ACM.

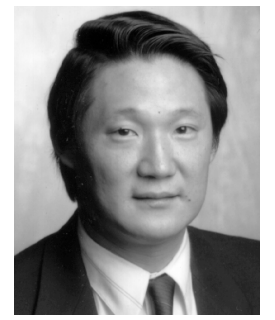

Philip S. Yu (S'76-M'78-SM'87-F'93) received the BS degree in electrical engineering from National Taiwan University, Taipei, Taiwan, Republic of China, in 1972, the MS and PhD degrees in electrical engineering from Stanford University in 1976 and 1978, respectively, and the MBA degree from New York University in 1982.

Since 1978, he has been with the IBM T.J. Watson Research Center, Yorktown Heights, New York. He is currently the manager of the Software Tools and Techniques Group in the Internet Technology Department. One of the focuses of the project is on the development of algorithms and tools for Internet applications, such as Web usage mining tool. His current research interests include database systems, data mining, Internet applications, multimedia systems, parallel and distributed processing, disk arrays, computer architecture, performance modeling, and workload analysis. Dr. Yu has published more than 200 papers in refereed journals and conferences, and more than 140 research reports and 90 invention disclosures. $\mathrm{He}$ holds or has applied for 42 U.S. patents.

Dr. Yu is a fellow of the ACM and the IEEE. He was an editor of IEEE Transactions on Knowledge and Data Engineering and also a guest coeditor of a special issue on mining databases. In addition to serving as a program committee member on various conferences, he was the program cochair of the Second International Workshop on Research Interests on Data Engineering: Transaction and Query Processing. He will be serving as the general chair of the 14th International Conference on Data Engineering. He has received several IBM and external honors, including Best Paper awards, two IBM Outstanding Innovation awards, Outstanding Technical Achievement award, research Division award, and 18 Invention Achievement awards. 